\title{
Single Antenna Target Detection Using Broadband Frequency Selection Time Reversal Method
}

\author{
Yi Jiang, Jian-Gang Zhu, Daniel D. Stancil, Jose Moura, Ahmet G. Cepni, \\ Benjamin Henty and Yuan-Wei Jin \\ Department of Electrical and Computer Engineering \\ Carnegie Mellon University, Pittsburgh, PA 15213
}

\section{Introduction}

In electromagnetic wave propagation, utilizing time reversal method, the super resolution focusing and background nulling has been experimentally demonstrated in highly cluttered environments [1][2]. Based on these time reversal concepts, a novel multiple-antenna detection scheme has been developed to substantially enhance the target detection in a highly scattering environment [3][4]. The spatial nullifying scheme significantly suppresses the reflection from scatterers and automatically enables wave energy focusing on the target.

In this paper, we present a broadband single antenna target detection scheme using frequency selection time reversal method to further improve the target detection in a cluttered environment. By re-transmitting time-reversed difference signal between reflections from the environment with and without the target, constructive interferences between the target and the background scatterers are utilized to enhance the return signal from target. Meanwhile, frequency selection time reversal emphasizes on the section of wavelengths with more constructive interferences and filters out the broadband white noise at other spectrum. Thus, signal to noise ratio (SNR) is substantially increased. The effectiveness of the proposed detection scheme has been demonstrated by numerical simulation. The simulation results show that using frequency selection time reversal method, the detection probability is significantly enhanced and SNR can be improved 2-5dB.

\section{Frequency Selection Time Reversal Detection Method}

Consider a single target in a highly cluttered environment shown in Figure 1. The broadband pulse in Figure 2(a) is broadcasted from a single transmitter to probe the environment. In the absence of target, the background channel response is given by $H_{w / o}(f)$ in Figure 2(b). When target comes into this environment, channel response changes to $H_{w}(f)$. The difference between these two channel responses is the target response, $H_{t}(f)$, given as $H_{t}(f)=H_{w /}(f)-H_{w / o}(f)$. The target response includes both reflections from target and scatterings between target and scatterers as shown in Figure 2(c). The target response is time-reversed and re-broadcasted into the environment. Mathematically, time reversal is phase conjugate in the frequency domain. Thus, the reflections from the target by retransmitting time reversal signal can be calculated by the following equation: $Y(f)=k H_{w /}(f) H_{t}^{*}(f)-k H_{w / o} H_{t}^{*}=k H_{t}(f) H_{t}^{*}(f) . k$ is the normalization factor 
chosen such that the energy of each retransmitted time reversal signal is maintained the same as the initially transmitted pulse.

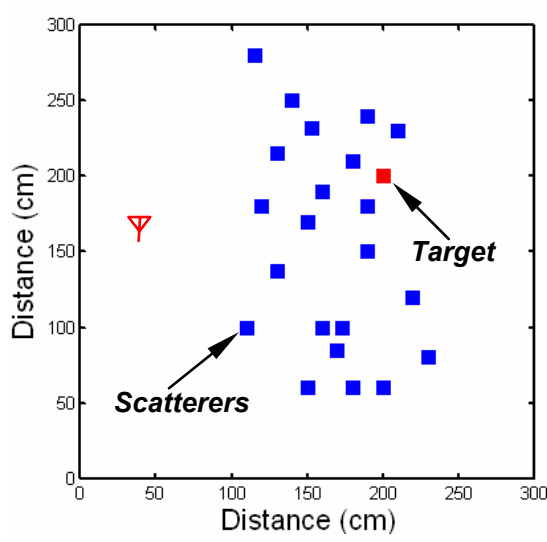

Figure 1 scatterers and target in a highly cluttered environment

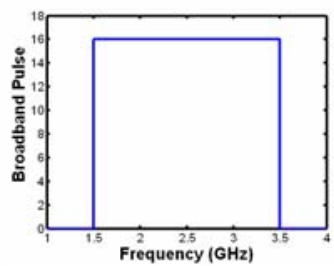

(a)

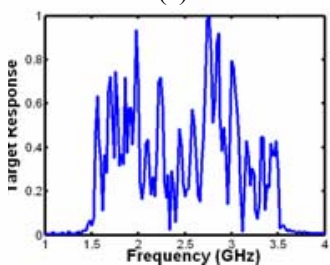

(c)

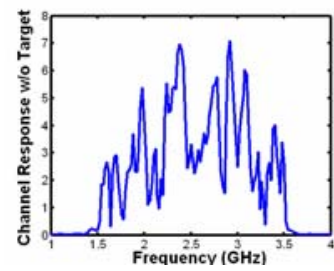

(b)

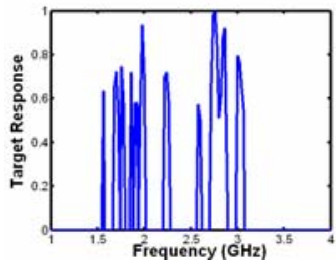

(d)

Figure 2 (a) Broadband Pulse (b) Reflections from field w/o Target $H_{w / o}(f)$ (c) Target Response $H_{t}(f)$ (d) Target Response $H_{t}^{\prime}(f)$ after frequency selection with cutoff $=0.5$

With the presence of broadband white noise, energy detector is used to assess different detection schemes. For a time reversal energy detector, energy of target return signal can be calculated as

$$
P_{t r d}=\int\left|Y(f)+N_{1}(f)\right|^{2} d f=\int\left|k H_{t}(f) H_{t}^{*}(f)+N_{1}(f)\right|^{2} d f
$$

Similarly, for change detection, energy of target response is given by

$$
P_{c d}=\int\left|H_{t}(f)+N_{2}(f)\right|^{2} d f
$$

$N_{l}(f)$ and $N_{2}(f)$ are additive white noises on the receiver. Detection decisions are made by comparing energies from time reversal detection and change detection signals with a chosen threshold.

Retransmitted time reversal difference signals focus on the target through interferences between the target and the environment. Thus, energy of $Y(f)$ is significantly bigger than the energy of $H_{t}(f)$. Peaks and valleys in the target response represent constructive and destructive interferences between target and background scatterers at different frequencies. A broadband signal is used here so that we can emphasis our wave energy on the frequencies with more constructive interferences. To achieve this idea, we normalize $H_{t}(f)$ with respect to its peak value and then cut the target response by a cutoff chosen between 0 and 1. Only the frequencies which have the normalized target response above the cutoff will be selected. A new target response, $H_{t}^{\prime}(f)$, with a cutoff at 0.5 , is shown in Figure 2(d). The energy for the frequency selection time reversal detection is given by:

$$
P_{f s t r d}=\int\left|Y^{\prime}(f)+N_{3}(f)\right|^{2} d f=\int\left|k^{\prime} H_{t}^{\prime}(f) H_{t}^{\prime^{*}}(f)+N_{3}(f)\right|^{2} d f
$$

After the frequency selection process, more energy focuses on the target due to stronger interferences between target and scatterers. Meanwhile, the wideband noise at the abandoned spectrum is filtered, thus, SNR is substantially increased. 


\section{Detection Results and Discussion}

Finite-difference-time-domain (FDTD) method has been utilized to simulate electromagnetic wave propagation for demonstration purpose. The simulation area is $3.6 \mathrm{~m} \times 3.6 \mathrm{~m}$ discretized by small square meshes with mesh size $1.2 \mathrm{~cm}$, one tenth of the wavelength of the carrier frequency. The probing pulse is a sinc pulse with $2 \mathrm{GHz}$ bandwidth and carrier frequency is $2.5 \mathrm{GHz}$.

Figure 3 shows the total return energy from the target for 1,000 runs for different detection schemes. From bottom to top, the five groups of dots represents received energy levels of pure white noise, change detection (CD), time reversal (TR), frequency selection time reversal with cutoff 0.5 (TR 0.5 ) and 0.8 (TR 0.8), respectively. The frequency selection time reversal increases the mean return energy from the target by a factor of 2.6 compared to change detection. Thus, SNR is increased by $4.1 \mathrm{~dB}$ in this case. Therefore, the detection probability can be substantially enhanced. This result shows the potential of time reversal for target detection in a highly cluttered environment.

Energy detector is used to assess the detection probabilities of different detection schemes when $S N R$ is $-10 d B$. SNR is defined by ratio of the energy between the change detection signal and the energy of the noise. Assume that white noise is only noise received on the receiving antenna. Knowing white noise energy level, we can set the threshold for detection by different false alarm rates (FAR). 1\% false alarm rate, shown in Figure 4, means that the probability of noise energy level above the threshold is $1 \%$. In another word, probability of the false detection caused by noise is $1 \%$. If energy of the received signal is above the threshold, it will be counted as one target detection. The probability density function is plotted as function of energy level in Figure 4 for different detection schemes. As shown in Figure 4, when noise energy level increases, frequency selection time reversal detection will have much better chance to detect the target than normal time reversal and change detection.

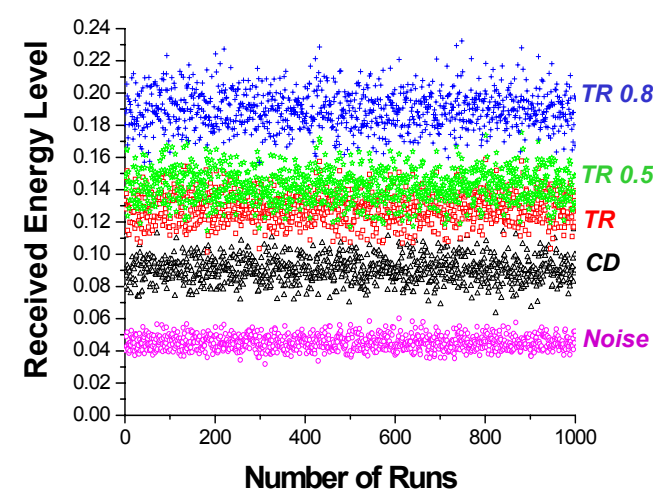

Figure 3 Target return energy levels at different cases for different detection schemes

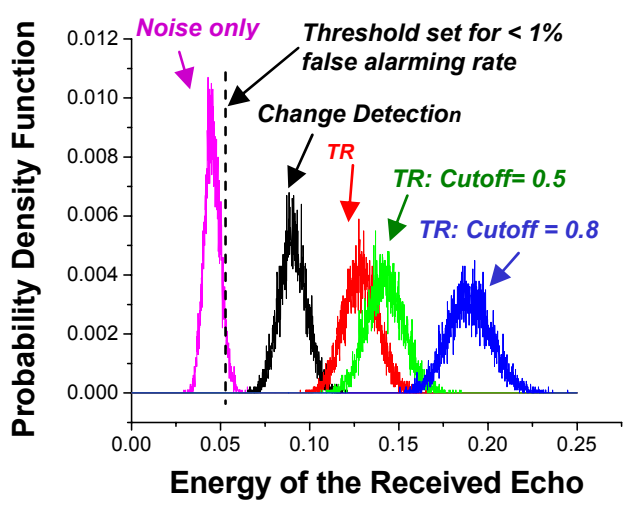

Figure 4 Probability distribution functions of the target return energy levels for different detection schemes 


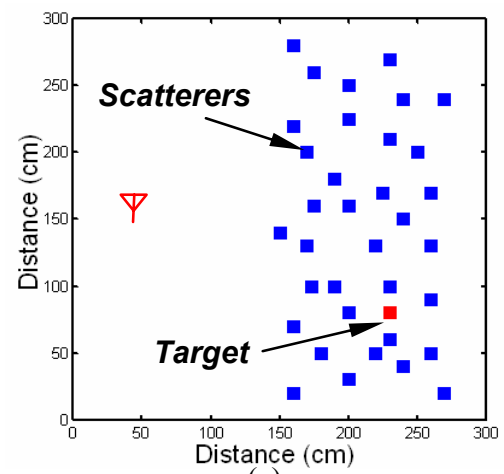

(a)

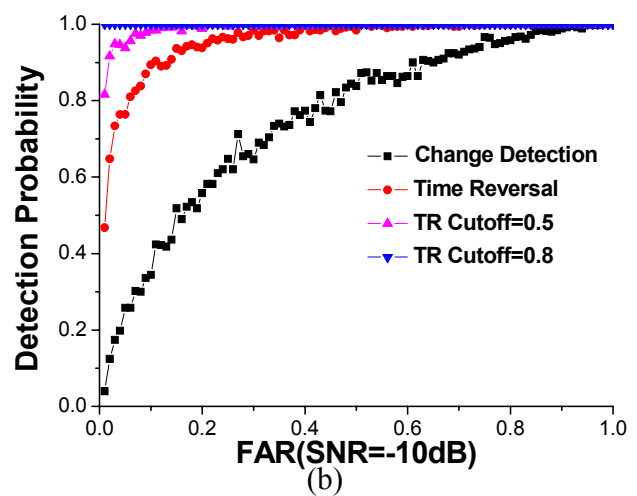

(b)

Figure 5 (a) Scatterers and target configuration (b) ROC Curve for different detection schemes

Figure 5 shows the receiving operating characteristics (ROC) curve for a 35scatterer detection case. It shows the detection probability for different detection schemes at different false alarm rate. For 1\% FAR, change detection almost fails at $\mathrm{SNR}=-10 \mathrm{~dB}$, while at the same time, time reversal detection can achieve around $45 \%$ detection probability and frequency selection time reversal detection can achieve almost $100 \%$ detection probability. For all other the different FAR, time reversal method significantly increases the detection probability compared to conventional change detection. Moreover, frequency selection time reversal further enhances the detection probability from time reversal method.

\section{Conclusion}

In this paper, a novel time reversal scheme utilizing broadband spectrum with a single antenna is presented. The scheme substantially enhances the probability for target detection in a highly cluttered environment with significant SNR gain. Narrow band selection within the utilized spectral range can further enhance the target detection ability. The rich multi-path environment gives time reversal scheme pronounced advantage over conventional radar detection schemes.

\section{References:}

[1] G. Lerosey, "Time reversal of electromagnetic waves", Physical Review letters, Vol. 92, No. 19, May 2004

[2] A.G. Cepni, D.D. Stancil, "Single antenna microwave nulling using time reversal techniques", Microwave Symposium Digest, IEEE MTT-S International, pp1723-1726, June2005

[3] J.G. Zhu, Y. Jiang, D. D. Stancil, Jose Moura, "A Novel Time Reversal for Target Detection in Cluttered Medium", IEEE Antenna and Propagation Symposium, vol. 4B pp. 135-138, July. 2005

[4] A. Cepni, D.D. Stancil, J.G. Zhu, Y. Jiang, "Experiment results on target detection in cluttered medium using time-reversal technique", IEEE Antenna and Propagation Symposium, vol. 4B pp. 139-142, July. 2005 